\title{
Hepatitis C infection and clearance: impact on atherosclerosis and cardiometabolic risk factors
}

Short title Hepatitis C, diabetes and atherosclerosis

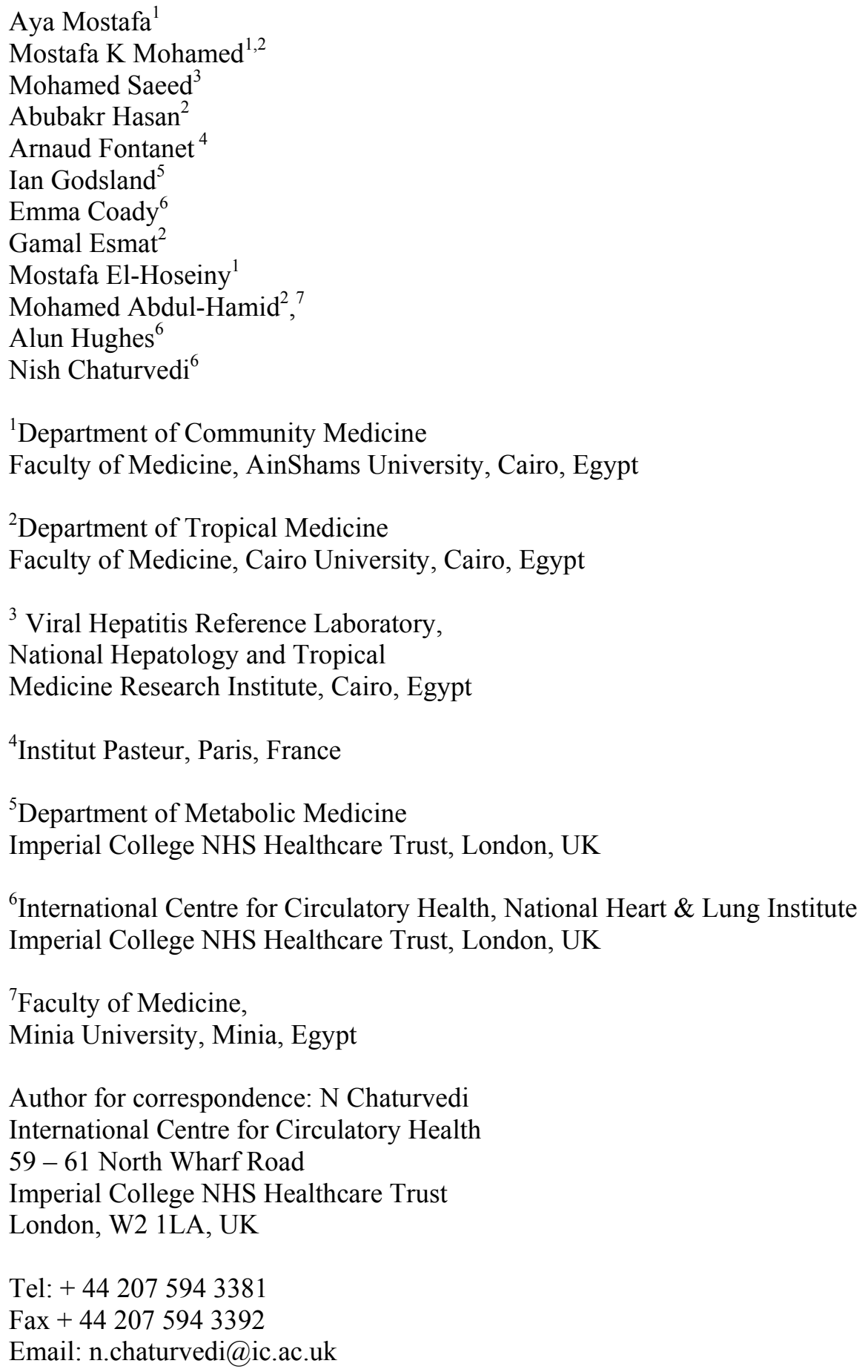

Word count : 3686 


\section{Abstract}

\section{Objective}

Chronic hepatitis $\mathrm{C}(\mathrm{HCV})$ infection is associated with diabetes and favourable lipids. We studied the effect of this paradox on atherosclerosis and cardiometabolic response to $\mathrm{HCV}$ clearance.

\section{Design \\ Cross-sectional study.}

\section{Setting}

Egypt.

\section{Participants}

329 chronically infected, 173 with cleared infection, and 795 never infected participants aged $\geq 35$ attended for baseline investigations. A subsample of 192, 115, and 187 respectively underwent ultrasound.

\section{Main outcome measures}

Diabetes, fasting glucose, lipids and fat deposition on ultrasound. Carotid intima media thickness (IMT) measured atherosclerosis.

\section{Results}

Diabetes prevalence was elevated $(10.1 \%(95 \%$ CI 6.6,13.6), $\mathrm{p}=0.04)$ in HCV chronic, and cleared $(10.1 \%(5.6,14.8), \mathrm{p}=0.08)$ individuals versus $6.6 \%(4.9,8.3)$ in those never infected. Mesenteric fat was elevated in chronic $(36.4 \mathrm{~mm}(34.5,38.2)), \mathrm{p}=0.004$, and cleared infection $(37.8(35.6,40.0))$, $\mathrm{p}<0.0001$ versus never infected $(32.7(31.0,34.4))$. LDL cholesterol was lower in chronic $(2.69$ $\mathrm{mmol} / 1(2.53,2.86) \mathrm{p}<0.001)$, but similar in cleared $(3.56(3.34,3.78) \mathrm{p}=0.4)$ versus never infected (3.45 (3.30,3.60)). Carotid IMT did not differ by infection status $(0.73(0.70,0.76, \mathrm{p}=0.4), 0.71$ $(0.66,0.75), \mathrm{p}=0.9), 0.71(0.68,0.74)$ respectively. Adjustment for cardiovascular risk factors elevated IMT in chronic infection $(0.76(0.72,0.79) \mathrm{p}=0.02)$, versus never infected individuals $(0.70$ $(0.67,0.73))$.

\section{Conclusions}

Hepatic function normalisation with HCV clearance may account for reversal of favourable lipids observed with HCV infection. Hyperglycaemia and visceral adiposity appear less amenable to HCV 
resolution. These different cardiovascular risk patterns may determine equivalent atherosclerosis risk by infection status. However, once these factors were accounted for, those with chronic infection had elevated IMT, suggesting a direct effect of infection.

Word count 247 


\section{Summary}

What is known about this subject?

1. Chronic HCV infection is associated with an elevated prevalence of diabetes and insulin resistance, yet lipid profiles are favourable, compared to people never infected with HCV.

2. Reports of the effect of this paradoxical risk factor profile on atherosclerosis are conflicting, due to limitations of previous study designs, either due to biased sampling, or lack of power.

3. The effect of HCV clearance on both diabetes and lipid profiles and downstream effect on atherosclerosis has not been studied.

What are the new findings?

1. Chronic HCV infection is associated with central fat deposition on ultrasound, in addition to the known elevated risk of diabetes and insulin resistance.

2. Atherosclerosis, as measured by intima media thickness (IMT) on carotid ultrasound was not elevated in those with chronic infection.

3. However, multivariate adjustment for cardiovascular risk factors revealed an elevated IMT in those with chronic infection, indicative of a direct effect of infection on atherosclerosis, masked by the effect of favourable lipid profiles.

4. Clearance of HCV infection was associated with reversal of favourable lipid profiles to those observed in never infected individuals. However, diabetes prevalence, and central obesity, remained elevated. No impact on carotid IMT was observed.

How might these findings impact on clinical practice?

1. As the developing world faces an epidemic of diabetes, consideration should be given to screening those with chronic or cleared HCV infection for early signs of diabetes, which should be managed accordingly.

2. Our findings provide reassurance that changes in cardiovascular risk factors in association with $\mathrm{HCV}$ infection do not result in elevated risks of atherosclerosis. 


\section{Introduction}

The developing world now faces the double burden of escalating rates of chronic, non-communicable disease on the background of endemic infection ${ }^{1}$. Important biological interactions may occur between these two groups of superficially unrelated conditions, specifically, cardiovascular disease (CVD) risk may be increased by infection ${ }^{2}$. Hepatitis $\mathrm{C}$ virus (HCV) infection is common in many parts of the developing world, and is associated with a 3.5 fold increased prevalence, and an over 10 fold increased incidence of type 2 diabetes ${ }^{3-5}$. This is likely to be a consequence of insulin resistance ${ }^{6}$. However, unlike the classical form of insulin resistance, chronic HCV infection is associated with a favourable lipoprotein profile ${ }^{7 ; 8}$, specifically reduced levels of apolipoprotein B (apoB)-containing lipoproteins, such as LDL and VLDL cholesterol ${ }^{9}$.

The consequence of this discordance between elevated diabetes prevalence, yet favourable lipoproteins, on CVD risk is unclear, but important to understand as CVD is now the major cause of death and disability worldwide. A single report indicates rates of coronary disease are elevated by $25 \%$ in those with chronic $\mathrm{HCV}$ infection ${ }^{10}$. But the more numerous studies measuring carotid intima media thickness (IMT), a valid proxy of atherosclerosis and future CVD risk ${ }^{11}$ have been conflicting $^{12-15}$. Discrepancies between studies are likely to be due to inadequacies in study design; for example, biased study population selection ${ }^{12}$, small numbers ${ }^{13}$, combining hepatitis $\mathrm{C}$ and $\mathrm{B}^{15}$, and biased comparator groups ${ }^{13}$, with inadequate adjustment for confounders. Further, no study has fully explored the impact of clearance of $\mathrm{HCV}$, either spontaneously or with treatment, on cardiometabolic risk.

Egypt has the highest HCV prevalence in the world (12\% prevalence of $\mathrm{HCV}$ antibody in the general population, $40 \%$ in persons over age 40 in rural areas $)^{16}$, and is a population that is susceptible to both diabetes and CVD. Egypt therefore is an ideal setting for a population based study, reducing biases introduced by studying selected clinic populations, and being more generalisable. Our aims were: 1. To confirm that chronic HCV infection is associated with both hyperglycaemia and a favourable lipoprotein profile compared to those never infected.

2. To determine how other aspects of the metabolic syndrome, specifically body fat distribution, blood pressure and insulin, differ by HCV infection status.

3. To compare carotid IMT, a measure of subclinical atherosclerosis, by HCV infection status, and explore the role of risk factors on IMT.

4. To determine the effect of HCV clearance on cardiometabolic risk. 


\section{Methods}

\section{Sample population}

Our study sampled from the village of Zawyat Razin, 200 miles from Cairo. A cohort study of the natural history of $\mathrm{HCV}$ was established in 2002. This recruited 4020 participants aged 3 to 88 years out of a total 5130 identified from a nominated sector of the village ${ }^{17}$. Of these 4020 individuals, 1143 were aged 35 years and above at the time of this study (199 with chronic HCV infection (positive anti-HCV antibodies and positive RNA), 127 with spontaneously cleared infection (positive anti-HCV antibodies and negative RNA) and 817 never infected by HCV (negative anti-HCV antibodies)). As risks of both diabetes and atherosclerosis increase with age, we chose to study adults only as we wished to be certain of detecting differences in cardiometabolic measures by infection group should they exist. To achieve the required sample size for the clinic study (see below), sampling was extended to the 845 individuals aged 35 years and above in a neighbouring sector of the village, who had participated in a voluntary HCV testing programme also in 2002 (208 with chronic $\mathrm{HCV}$ infection, 120 with spontaneously cleared infection, and 517 never infected). Disease distribution in the voluntary testing programme was similar to that in the HCV cohort study. This yielded a total sample population of 1988 .

\section{Village metabolic study}

These 1988 individuals were invited to take part in the village metabolic study. After informed consent, a questionnaire collecting demographic, medical and lifestyle data was administered, and the following measurements were performed: height and weight, waist (midway between the umbilicus and anterior superior iliac spine) and hip (at the level of the greater trochanter) circumference, seated blood pressure calculated as the average of three measurement after 10 minutes rest using a validated oscillometric device (Omron 705CP), and fasting blood samples were collected for measurement of glucose and lipid profiles. These samples were sent to Cairo on a daily basis for analysis.

\section{Clinic cardiometabolic study}

All those attending the village metabolic study were eligible for a detailed clinic examination for cardiometabolic status at the National Hepatology and Tropical Medicine Research Institute in Cairo. The target was 210 attendees each in the chronic, cleared and never infected categories. The three infection status groups were stratified into 5 year age bands, and similar numbers of individuals from each band for those with chronic or cleared infection (where numbers were most limited) were first invited to the clinic. We had hoped to match by sex in each age stratum but numbers in each cell were too limited. Recruitment for those with chronic infection was completed first, and numbers in 
requisite age strata for cleared infection were exhausted at 135 recruits. Recruiting beyond this number would have seriously unmatched the age distribution of this group, making analysis difficult. Substantially more of those never infected agreed to participate than required, so transfer to the clinic study was stopped when the requisite sample was completed.

During the Cairo clinic visit anthropometric measurements (weight and height, waist and hip circumference) were again performed, and in addition, measurement of bioimpedence for total percentage of body fat (Tanita TBF-300M), and abdominal ultrasound using a Siemens Energia machine, to assess regional fat distribution, specifically mesenteric, (3-5 MHz curved transducer in the para-umbilical area, to identify mesenteric leaves, calculating the mean of the thickest 3 leaves) and abdominal subcutaneous fat (linear 7-12 MHZ transducer at L5, again the mean of 3 measures taken of the maximum thickness), all as previously described ${ }^{18}$. Clinic sitting blood pressure was again measured using a validated oscillometric device (Omron 705CP).

Carotid ultrasound measurements with concurrent ECG recording were performed using a Philips HDI ATL 5000 ultrasound machine equipped with a linear array broad band 7-4 MHz transducer. Carotid IMT was measured in the far wall of the left common carotid artery (CCA) and carotid bulb ${ }^{19}$. B mode images were saved as cineloops and measurement of IMT and lumen diameter was performed off-line from images acquired in diastole (identified by the ECG R wave) using a validated semiautomatic program ${ }^{20}$. Atherosclerotic plaques (defined as focal thickenings of the intima-media $>1.3 \mathrm{~mm}$ or a distinct area with an IMT $>50 \%$ thicker than the adjacent region) were imaged using B mode ultrasound using colour or power Doppler to assist visualization if necessary. A single observer made all ultrasound measurements blinded to patient status.

A $30 \mathrm{ml}$ fasting blood sample was drawn, and sent locally for measurement of AST, ALT, $\gamma \mathrm{GT}$, albumin, total bilirubin, lipid profile (fasting serum cholesterol, HDL cholesterol, triglycerides, LDL (Friedewald method)) and glucose, all using standard methods on a Beckman Synchron CX4 Clinical Analyser (Beckman Instruments, Brea, CA, USA). Insulin was determined using AxSYM Insulin Assay (Abbott Laboratories, Wiesbaden Germany), and C-peptide was determined using DRG Cpeptide ELISA kit (Marburg, Germany) according to manufacturer instructions. The diagnosis of HCV infection was confirmed by serology testing using an Elisa (INNOTEST® HCV Ab IV, Innogenetics, Ghent, Belgium) and, when positive, confirmed by Abbott HCV EIA 3.0 (Abbott Laboratories, Diagnostics Division, IL, USA). Molecular identification of HCV RNA was performed using a one-step reverse transcriptase-polymerase chain reaction (RT-PCR) assay. Individuals testing 
positive by the two serological tests and PCR positive were considered as HCV positive, with chronic infection if HCV RNA was positive. Individuals with negative serological results were considered as never infected by HCV.

\section{Sample size and statistical analysis}

The main study outcome was mean carotid IMT. We required 190 participants per group to show a standardised difference of 0.29 between those with chronic versus no $\mathrm{HCV}$ infection (alpha $=0.05$, power $=0.8$, two-sided tests). This means that if we assume a population standard deviation in carotid IMT of $0.18 \mathrm{~mm}$, we could detect a difference in mean IMT between infection groups of at least 0.05 $\mathrm{mm}$. No study has reported mean values of carotid IMT between those with and without HCV, although a nearly 3 fold increase in risk of IMT thickening above a given cutpoint has been reported $^{12}$. Previous studies of the association between IMT and the presence or absence of chlamydia pneumoniae report a standardised difference of $0.40^{21}$. We wished to ensure that a difference of at least this magnitude could be detected here, and settled for the more conservative effect of 0.29 , anything larger could then be detected with much greater power. A total of 210 attendees in each group were required to allow for missing data.

Variables not normally distributed were log transformed for analysis. Mean values for normally distributed and transformed data, with $95 \%$ confidence limits, were estimated using regression analysis. As the comparison groups differed in age and sex distribution, age and sex adjusted values are presented throughout although in practice this made modest differences to the estimates. For categorical variables, direct standardization for age and sex was performed, using the total study population (be it at the village or in the Cairo clinic), as the standard. Pearson (for normally distributed data), and Spearman (for non-parametric data) correlation coefficients between key variables were estimated. Multivariate regression analysis for continuous variables was performed using analysis of co-variance, and for categorical variables logistic regression, with adjustment for variables that biologically were thought to act as confounders. Age and sex were forced into these models. Thus any residual differences between infection categories are unlikely to be due to imbalances in the key immutable characteristics of age and sex, allowing us to focus on reversible risk factors and provide insights into mechanisms.

The ADA definition of diabetes was used ${ }^{22}$. Hypertension was defined as either being on self reported medication for hypertension, or systolic pressure $\geq 140 \mathrm{mmHg}$, or diastolic pressure $\geq 90$ $\mathrm{mmHg}$. 


\section{Results}

Village study of diabetes prevalence and lipid profiles

A total of 795 (60\% response rate) people who had never been infected with HCV, 173 (70\% response rate) with cleared infection, and 329 (81\% response rate) with chronic infection were examined in the village (overall $65 \%$ response rate). Those with chronic infection or cleared infection were older and more likely to be male than those never infected (table 1). However those with chronic infection, although lighter, had elevated fasting glucose, and elevated prevalence of diabetes (odds ratio $1.31,95 \%$ CI 1.03,1.66, $\mathrm{p}=0.03$, adjusted for age and sex) than never infected individuals. Further adjustment for BMI did not alter this excess risk (odds ratio 1.35, 95\% CI $1.06,1.73, \mathrm{p}=0.02$ ). In contrast, lipid profiles in those with chronic infection were favourable, with lower total and LDL cholesterol, and lower triglyceride.

Clearance of infection was associated with persistent elevation of diabetes prevalence, but a fasting glucose that was midway between those never infected and those with chronic infection. Weight, lipid and lipoprotein profiles are similar to those observed in never infected individuals.

\section{Clinic study of IMT and fat imaging}

A subgroup of 227 never infected individuals, 135 with cleared infection, and 207 with chronic infection attended the clinic in Cairo for IMT scans. All those with cleared and chronic infection studied in the village were invited to yield sufficient numbers, a response rate of $78 \%$ and $63 \%$ respectively. Quota sampling was used to obtain the 227 who had never been infected, as many more volunteered than were required in this category.

Analysable IMT data were successfully obtained on 192 never infected, 115 with cleared infection, and 187 with chronic infection (missing data was due to limited access to equipment, equipment breakdown, and scans of insufficient quality). Differences observed in the village population in demographic and metabolic characteristics by infection status were also observed in the clinic sample, although some of the associations did not reach statistical significance (table 2). For example, the odds ratio for diabetes comparing chronic with never infected individuals, at 1.26, was similar to that observed in the village, but was not statistically significant due to the smaller numbers studied here $((95 \%$ CI $0.82,1.95), \mathrm{p}=0.3$ adjusted for age and sex $)$. Among the additional measurements made in the clinic, fasting insulin and HOMA-IR were both significantly higher in those with chronic infection than in those never infected or those with cleared infection. 
BMI, and total \%fat were lower in those with chronic infection, than in those with cleared or no infection. Although no differences in waist to hip ratio by infection status were observed, mesenteric fat levels were elevated in those with chronic infection, whilst subcutaneous fat levels were reduced, compared to those never infected. Clearance of infection was associated with BMI and fat $\%$ equivalent to that of never infected individuals, whilst mesenteric fat remained elevated.

Age and sex adjusted mean carotid IMT and proportion of individuals with carotid plaque did not differ significantly by infection status (table 3 ). Adjusting for all CVD risk factors, whether favourable or not, resulted in a carotid IMT, and burden of plaque, that was now significantly higher in those with chronic versus never infection. For plaque, it should be noted that numbers were small, resulting in instability of estimates when subject to multivariate adjustment. The major contributors to the multivariate model for IMT were LDL cholesterol $(\mathrm{p}=0.002)$, and systolic blood pressure $(p=0.008)$. 


\section{Discussion}

We confirm that people with chronic HCV infection have an elevated prevalence of diabetes, are more likely to be hyperglycaemic, and insulin resistant, compared to those who were never infected, even though they are on average lighter with less total fat mass. Despite the insulin resistance, lipid and lipoprotein profiles are favourable. Novel findings from this study include the observation that chronic infection is associated with central fat deposition, and loss of subcutaneous fat. Secondly, we show that clearance of infection was associated with resolution of lipid and lipoprotein profiles, and total weight approximating never infected individuals, but persistent, albeit non-significant elevations in glucose and diabetes prevalence, and persistence of central fat deposition. Thirdly, we demonstrate that carotid IMT, a measure of subclinical atherosclerosis, did not differ significantly by infection status. Adjustment for conventional CVD risk factors, particularly LDL cholesterol and systolic blood pressure, resulted in an IMT in those with chronic infection that was significantly elevated compared to those never infected. Findings for carotid plaque, although numbers were small, confirmed those for mean IMT. This may suggest a direct effect of infection on atherosclerosis.

We show that diabetes risk is elevated by about a third in association with chronic HCV infection. Previous studies report much greater risks, but have important limitations which may exaggerate risks. These include restriction of $\mathrm{HCV}$ cases to those with cirrhotic disease ${ }^{5}$, small numbers ${ }^{3}$, a comparator population made up of cases with hepatitis B and other liver disorders ${ }^{23}$, or a healthy population sample drawn from a very different source to cases ${ }^{24}$. In many countries, HCV infection is commonly associated with drug abuse; to draw a comparison with individuals participating in national health surveys, or attending clinics and hospitals for other reasons, is vulnerable to bias. Others have matched cases (with HCV) and controls (without HCV) for a number of risk factors, including obesity ${ }^{25}$. According to our data, people with chronic HCV in the population are lean, thus matching for obesity status will result in recruiting very lean individuals from the control population, who would have very low risks of diabetes, thus exaggerating the risk of diabetes associated with infection. Interestingly, reports of comparisons within a healthy population based health survey have been conflicting, with one reporting an increased prevalence of diabetes associated with $\mathrm{HCV}^{4}$, and the other not $^{26}$. As the HCV epidemic in Egypt is to some extent a consequence of a population wide schistosomiasis treatment programme undertaken in the 1960s, sampling from a village population, who were all exposed to identical conditions, is a more optimal sampling strategy.

Despite diabetes and insulin resistance, we confirm the very specific lipid and lipoprotein profile in chronic HCV infection, predominantly affecting total and LDL cholesterol ${ }^{9 ; 26}$. Derangements in lipid 
metabolism have been proposed to be due to a variety of mechanisms including impaired assembly and secretion of VLDL as a consequence of the effect of $\mathrm{HCV}$ core protein on microsomal transfer protein, alterations in the composition of VLDL, and alterations in VLDL and LDL catabolism ${ }^{27228}$. All these effects occur in the liver, thus clearance of HCV infection, which resolves hepatic damage, should also resolve the lipoprotein changes. This is what we see in the group of individuals who had cleared their infection. That resolution of $\mathrm{HCV}$, and presumably to some extent liver damage, does not affect diabetes prevalence or unfavourable ectopic fat distribution, and does not wholly reduce the hyperglycaemia associated with chronic infection, suggests that other mechanisms are involved in these changes, or that these effects of infection are resistant to change. In the case of diabetes itself, it is likely that once an individual has this diagnostic label they are unlikely to lose it even if glucose levels return to normal, thus prevalence estimates will often lag behind actual changes in glucose. Of course glycaemic and lipoprotein profiles are influenced by lifestyle factors such as diet and exercise, and we do not have precise measures of these to determine their role. Yet whilst these lifestyle factors may differ by infection status, it would be hard to hypothesise a dietary and exercise pattern that results in diabetes on the one hand, yet favourable lipoprotein patterns on the other. Thus we do not believe our findings are due to residual confounding. These metabolic changes were observed in individuals who cleared infection spontaneously; data on the impact of antiviral therapy on metabolic characteristics are conflicting, and require further investigation ${ }^{29 ; 30}$

Our second novel finding, that chronic HCV infection is associated with low total body fat, but increased deposition of fat around the viscera, whilst subcutaneous fat deposition is reduced, is consistent with the greater degree of insulin resistance and diabetes in this group, but somewhat at odds with previous studies, although many matched for obesity status so these previous comparisons are prone to bias. Studies unmatched for obesity however suggest that HCV patients are more obese overall, and had greater waist circumferences than controls ${ }^{31}$. However controls in this case were selected from hospital staff members and relatives, and again, are not representative of the source population of cases. An analysis of a population based health survey (where clearly cases will come from the same source population as non-cases) does support our finding of less obesity, and narrower waist circumference in the presence of chronic infection, at least in those aged 20-59 years ${ }^{26}$. A narrower waist circumference in tandem with ultrasound evidence of visceral fat deposition is not inconsistent, as waist circumference includes both visceral and subcutaneous fat. As subcutaneous fat levels are lower in those with chronic infection, it is likely that loss from this fat depot contributes to the lower waist circumference. 
Our finding of carotid IMT levels that did not differ significantly by infection status is again in contrast to some previous reports. Again there are concerns regarding the comparisons made in previous studies, with controls recruited from hospital staff ${ }^{31}$, cases being a mixture of individuals with hepatitis $\mathrm{B}$ and $\mathrm{C}^{15}$, and comparisons made between angiography patients with and without diagnosed coronary disease ${ }^{13}$. A comparison within a group attending for health screening found markedly more plaque (IMT $>1.3 \mathrm{~mm}$ ) and more carotid intima-medial thickening (IMT $\geq 1 \mathrm{~mm}$ ) in those who were $\mathrm{HCV}$ positive, but these individuals were on average 4 years older than non-infected persons, and mean carotid IMT, (our key outcome), did not differ significantly ${ }^{12 ; 32}$.

We conclude that hyperglycaemia and insulin resistance on the one hand, and a favourable lipid and lipoprotein profile on the other, results in a degree of atherosclerosis in people with chronic HCV infection that is no different to those never infected. We could not measure an emerging important component of the metabolic syndrome, non-alcoholic fatty liver disease (NAFLD), which is a strong independent predictor of cardiovascular disease ${ }^{33}$, as this would have required biopsy for accuracy. However, given the different balance of existing risk factors associated with NAFLD, it is unclear whether its prevalence would be higher, lower or about the same in those with chronic HCV infection versus those never infected. An elevated IMT in those with chronic infection emerges when accounting for CVD risk factors, suggesting that there may be a direct effect of infection on subclinical atherosclerosis. This observation confirms the single large report of an elevated risk of coronary events in those with chronic infection ${ }^{10}$, only noted on multivariate adjustment, even though individuals with chronic HCV had a generally more favourable CVD risk factor profile, forcing the authors to conclude that excess CVD risk in those with chronic HCV infection must occur by nonconventional pathways, associated with the infection itself. Clearance of infection returns lipoprotein profiles to normal levels, perhaps as a consequence of resolution of liver damage, but does not remove the excess diabetes risk, nor the central fat deposition, suggesting that mechanisms for these disturbances are different, or that they are less amenable to reversal as a consequence of treatment. Although we show no excess carotid IMT in these individuals, long term follow up data are required to determine the impact of infection clearance on atherosclerosis, particularly given the reversal of favourable lipoprotein profiles. As the burden of CVD increases in these populations, and as attempts focus on treating HCV infection worldwide, this question requires urgent attention. 


\section{Acknowledgements}

Authors at Imperial College acknowledge support of the BRC.

\section{Funding}

This project was supported by a project grant from the Wellcome Trust [078180/Z/05/Z].

\section{Licence for Publication}

The Corresponding Author has the right to grant on behalf of all authors and does grant on behalf of all authors, an exclusive licence (or non exclusive for government employees) on a worldwide basis to the BMJ Publishing Group Ltd and its Licensees to permit this article (if accepted) to be published in Gut editions and any other BMJPGL products to exploit all subsidiary rights, as set out in our licence(http://group.bmj.com/products/journals/instructions-forauthors/licence-forms).

\section{Competing Interest}

None to declare for all authors. 


\section{References}

1. Lopez AD, Mathers CD, Ezzati M, Jamison DT, Murray CJ: Global and regional burden of disease and risk factors, 2001: systematic analysis of population health data. Lancet $367: 1747-1757,2006$

2. Smeeth L, Thomas SL, Hall AJ, Hubbard R, Farrington P, Vallance P: Risk of myocardial infarction and stroke after acute infection or vaccination. $N$ Engl J Med 351:2611-2618, 2004

3. Mehta SH, Brancati FL, Strathdee SA, Pankow JS, Netski D, Coresh J, Szklo M, Thomas DL: Hepatitis $C$ virus infection and incident type 2 diabetes. Hepatology 38:50-56, 2003

4. Mehta SH, Brancati FL, Sulkowski MS, Strathdee SA, Szklo M, Thomas DL: Prevalence of type 2 diabetes mellitus among persons with hepatitis $\mathrm{C}$ virus infection in the United States. Ann Intern Med 133:592-599, 2000

5. Allison ME, Wreghitt T, Palmer CR, Alexander GJ: Evidence for a link between hepatitis C virus infection and diabetes mellitus in a cirrhotic population. J Hepatol 21:1135-1139, 1994

6. Yazicioglu G, Isitan F, Altunbas H, Suleymanlar I, Ozdogan M, Balci MK, Karayalcin U: Insulin resistance in chronic hepatitis C. Int J Clin Pract 58:1020-1022, 2004

7. Dai CY, Chuang WL, Ho CK, Hsieh MY, Huang JF, Lee LP, Hou NJ, Lin ZY, Chen SC, Hsieh MY, Wang LY, Tsai JF, Chang WY, Yu ML: Associations between hepatitis C viremia and low serum triglyceride and cholesterol levels: a community-based study. $J$ Hepatol 49:9-16, 2008

8. Marzouk D, Sass J, Bakr I, El HM, bdel-Hamid M, Rekacewicz C, Chaturvedi N, Mohamed $\mathrm{MK}$, Fontanet A: Metabolic and cardiovascular risk profiles and hepatitis $\mathrm{C}$ virus infection in rural Egypt. Gut 56:1105-1110, 2007

9. Serfaty L, Andreani T, Giral P, Carbonell N, Chazouilleres O, Poupon R: Hepatitis C virus induced hypobetalipoproteinemia: a possible mechanism for steatosis in chronic hepatitis C. $J$ Hepatol 34:428-434, 2001

10. Butt AA, Xiaoqiang W, Budoff M, Leaf D, Kuller LH, Justice AC: Hepatitis C virus infection and the risk of coronary disease. Clin Infect Dis 49:225-232, 2009

11. O'Leary DH, Polak JF, Kronmal RA, Manolio TA, Burke GL, Wolfson SKJ: Carotid-artery intima and media thickness as a risk factor for myocardial infarction and stroke in older adults. Cardiovascular Health Study Collaborative Research Group [see comments]. $N$ Engl $J$ Med 340:14-22, 1999

12. Ishizaka $\mathrm{N}$, Ishizaka $\mathrm{Y}$, Takahashi $\mathrm{E}$, Tooda $\mathrm{E}$, Hashimoto H, Nagai R, Yamakado M: Association between hepatitis $\mathrm{C}$ virus seropositivity, carotid-artery plaque, and intima-media thickening. Lancet 359:133-135, 2002

13. Vassalle C, Masini S, Bianchi F, Zucchelli GC: Evidence for association between hepatitis C virus seropositivity and coronary artery disease. Heart 90:565-566, 2004 
14. Bilora F, Rinaldi R, Boccioletti V, Petrobelli F, Girolami A: Chronic viral hepatitis: a prospective factor against atherosclerosis. A study with echo-color Doppler of the carotid and femoral arteries and the abdominal aorta. Gastroenterol Clin Biol 26:1001-1004, 2002

15. Volzke H, Schwahn C, Wolff B, Mentel R, Robinson DM, Kleine V, Felix SB, John U: Hepatitis $\mathrm{B}$ and $\mathrm{C}$ virus infection and the risk of atherosclerosis in a general population. Atheroscler 174:99-103, 2004

16. Habib M, Mohamed MK, bdel-Aziz F, Magder LS, bdel-Hamid M, Gamil F, Madkour S, Mikhail NN, Anwar W, Strickland GT, Fix AD, Sallam I: Hepatitis C virus infection in a community in the Nile Delta: risk factors for seropositivity. Hepatology 33:248-253, 2001

17. Arafa N, El Hoseiny M, Rekacewicz C, Bakr I, El-Kafrawy S, El Daly M, Aoun S, Marzouk D, Mohamed MK, Fontanet A: Changing pattern of HCV spread in rural areas of Egypt. $J$ Hepatol 43:418-424, 2005

18. Liu KH, Chan YL, Chan WB, Kong WL, Kong MO, Chan JC: Sonographic measurement of mesenteric fat thickness is a good correlate with cardiovascular risk factors: comparison with subcutaneous and preperitoneal fat thickness, magnetic resonance imaging and anthropometric indexes. Int J Obes Relat Metab Disord 27:1267-1273, 2003

19. Wendelhag I, Wiklund O, Wikstrand J: On quantifying plaque size and intima-media thickness in carotid and femoral arteries. Comments on results from a prospective ultrasound study in patients with familial hypercholesterolemia. Arterioscler Thromb Vasc Biol 16:843850,1996

20. Wendelhag I, Liang Q, Gustavsson T, Wikstrand J: A new automated computerized analyzing system simplifies readings and reduces the variability in ultrasound measurement of intimamedia thickness. Stroke 28:2195-2200, 1997

21. Schmidt C, Hulthe J, Wikstrand J, Gnarpe H, Gnarpe J, Agewall S, Fagerberg B: Chlamydia pneumoniae seropositivity is associated with carotid artery intima-media thickness. Stroke 31:1526-1531, 2000

22. American Diabetes Association: Report of the expert committee on the diagnosis and classification of diabetes mellitus. Diabetes Care 20:1183-1197, 1997

23. Grimbert S, Valensi P, Levy-Marchal C, Perret G, Richardet JP, Raffoux C, Trinchet JC, Beaugrand M: High prevalence of diabetes mellitus in patients with chronic hepatitis C. A case-control study. Gastroenterol Clin Biol 20:544-548, 1996

24. Zein CO, Levy C, Basu A, Zein NN: Chronic hepatitis C and type II diabetes mellitus: a prospective cross-sectional study. Am J Gastroenterol 100:48-55, 2005

25. Knobler H, Schihmanter R, Zifroni A, Fenakel G, Schattner A: Increased risk of type 2 diabetes in noncirrhotic patients with chronic hepatitis $\mathrm{C}$ virus infection. Mayo Clin Proc 75:355-359, 2000

26. Behrendt CE, Ruiz RB: Hyperglycemia among persons with hepatitis C: not the classical diabetic phenotype. Diabetes Res Clin Pract 71:68-74, 2006 
27. Perlemuter G, Sabile A, Letteron P, Vona G, Topilco A, Chretien Y, Koike K, Pessayre D, Chapman J, Barba G, Brechot C: Hepatitis C virus core protein inhibits microsomal triglyceride transfer protein activity and very low density lipoprotein secretion: a model of viral-related steatosis. FASEB J 16:185-194, 2002

28. Napolitano M, Giuliani A, Alonzi T, Mancone C, D'Offizi G, Tripodi M, Bravo E: Very low density lipoprotein and low density lipoprotein isolated from patients with hepatitis $\mathrm{C}$ infection induce altered cellular lipid metabolism. J Med Virol 79:254-258, 2007

29. Giordanino C, Bugianesi E, Smedile A, Ciancio A, Abate ML, Olivero A, Pellicano R, Cassader M, Gambino R, Bo S, Ciccone G, Rizzetto M, Saracco G: Incidence of type 2 diabetes mellitus and glucose abnormalities in patients with chronic hepatitis $\mathrm{C}$ infection by response to treatment: results of a cohort study. Am J Gastroenterol 103:2481-2487, 2008

30. Kawaguchi T, Ide T, Taniguchi E, Hirano E, Itou M, Sumie S, Nagao Y, Yanagimoto C, Hanada S, Koga H, Sata M: Clearance of HCV improves insulin resistance, beta-cell function, and hepatic expression of insulin receptor substrate 1 and 2. Am J Gastroenterol 102:570-576, 2007

31. Targher G, Bertolini L, Padovani R, Rodella S, Arcaro G, Day C: Differences and similarities in early atherosclerosis between patients with non-alcoholic steatohepatitis and chronic hepatitis B and C. J Hepatol 46:1126-1132, 2007

32. Ishizaka Y, Ishizaka N, Takahashi E, Unuma T, Tooda E, Hashimoto H, Nagai R, Yamakado M: Association between hepatitis $\mathrm{C}$ virus core protein and carotid atherosclerosis. Circ $J$ 67:26-30, 2003

33. Dunn W, Xu R, Wingard DL, Rogers C, Angulo P, Younossi ZM, Schwimmer JB: Suspected nonalcoholic fatty liver disease and mortality risk in a population-based cohort study. Am J Gastroenterol 103:2263-2271, 2008 
Table 1. Demographic and metabolic characteristics by infection status of individuals studied in the village.

\begin{tabular}{|c|c|c|c|c|c|c|c|c|}
\hline \multirow[b]{2}{*}{ Variable } & \multicolumn{2}{|c|}{ Never infected $(n=795)$} & \multicolumn{3}{|c|}{ Cleared infection $(n=173)$} & \multicolumn{3}{|c|}{ Chronic infection ( $n=329)$} \\
\hline & Mean or $\%$ & $95 \% \mathrm{CI}$ & Mean or $\%$ & $95 \% \mathrm{CI}$ & $\mathrm{p}$ value & Mean or \% & $95 \% \mathrm{CI}$ & $\mathrm{p}$ value \\
\hline Age (years) & 49.4 & $48.6,50.1$ & 51.4 & $49.9,52.9$ & 0.01 & 51.0 & $50.0,51.9$ & 0.01 \\
\hline Male \% (n) & $37(295)$ & & $49(85)$ & & 0.003 & $59(195)$ & & $<0.0001$ \\
\hline BMI, $\mathrm{kg} / \mathrm{m}^{2}$ & 29.4 & $29.0,29.8$ & 29.5 & $28.6,30.3$ & 0.8 & 28.0 & $27.4,28.6$ & $<0.0001$ \\
\hline Waist hip ratio (men) & 0.91 & $0.90,0.92$ & 0.92 & $0.91,0.94$ & 0.3 & 0.92 & $0.91,0.93$ & 0.6 \\
\hline Waist hip ratio (women) & 0.87 & $0.86,0.88$ & 0.86 & $0.85,0.88$ & 0.2 & 0.87 & $0.86,0.88$ & 0.4 \\
\hline SBP, mmHg & 123 & 121,124 & 120 & 117,123 & 0.07 & 121 & 118,123 & 0.08 \\
\hline $\mathrm{DBP}, \mathrm{mmHg}$ & 75 & 74,76 & 73 & 71,75 & 0.05 & 74 & 73,75 & 0.3 \\
\hline Total cholesterol, mmol/1 & 5.19 & $5.11,5.28$ & 5.16 & $4.98,5.34$ & 0.8 & 4.33 & $4.20,4.46$ & $<0.0001$ \\
\hline LDL-cholesterol, mmol/1 & 3.40 & $3.32,3.47$ & 3.43 & $3.25,3.60$ & 0.8 & 2.67 & $2.55,2.80$ & $<\mathbf{0 . 0 0 0 1}$ \\
\hline HDL-cholesterol, mmol/1 & 1.24 & $1.20,1.28$ & 1.16 & $1.07,1.26$ & 0.2 & 1.18 & $1.11,1.25$ & 0.2 \\
\hline Triglyceride, $\mathrm{mmol} / \mathrm{l}^{*}$ & 1.10 & $1.06,1.15$ & 1.16 & $1.06,1.26$ & 0.4 & 0.97 & $0.91,1.03$ & 0.001 \\
\hline Glucose, $\mathrm{mmol} / \mathrm{l}^{*}$ & 4.83 & $4.74,4.90$ & 4.95 & $4.76,5.15$ & 0.3 & 5.05 & $4.90,5.20$ & 0.009 \\
\hline Albumin, $\mathrm{g} / 1$ & 38.3 & $38.0,38.6$ & 38.3 & $37.7,39.0$ & 0.9 & 37.7 & $37.2,38.1$ & 0.02 \\
\hline $\mathrm{ALT}, \mathrm{IU} / \mathrm{L}^{*}$ & 19.6 & $19.0,20.3$ & 19.1 & $17.8,20.4$ & 0.5 & 35.5 & $33.8,37.3$ & $<0.0001$ \\
\hline $\mathrm{AST}, \mathrm{IU} / \mathrm{L}^{*}$ & 20.3 & $19.6,20.9$ & 20.4 & $19.1,21.8$ & 0.8 & 36.7 & $34.8,38.5$ & $<0.0001$ \\
\hline$\gamma \mathrm{GT}, \mathrm{IU} / \mathrm{L}^{*}$ & 26.8 & $23.1,31.2$ & 22.2 & $18.2,27.1$ & 0.1 & 27.1 & $22.2,32.3$ & 0.9 \\
\hline Total bilirubin, $\mu \mathrm{mol} / \mathrm{I}^{*}$ & 10.1 & $9.8,10.4$ & 10.9 & $10.2,11.7$ & 0.05 & 12.8 & $12.1,13.4$ & $<0.0001$ \\
\hline Diabetes & 6.6 & $4.9,8.3$ & 10.1 & $5.6,14.8$ & 0.08 & 10.1 & $6.6,13.6$ & 0.04 \\
\hline Hypertension & 14.3 & $11.9,16.8$ & 17.2 & $11.8,22.5$ & 0.3 & 17.2 & $12.8,21.5$ & 0.2 \\
\hline Current smoker & 8.7 & $6.7,10.7$ & 8.9 & 4.4,13.4 & 1.0 & 11.1 & $7.7,14.7$ & 0.2 \\
\hline
\end{tabular}

Apart from age and sex themselves, all other parameters are adjusted (continuous) or standardised (categorical) for age and sex (except waist hip ratio, where age adjusted values are presented stratified by sex). $\mathrm{P}$ values comparing either cleared, or chronic infection, with no infection.

*Log transformed variable, geometric means reported.

Abbreviations: ALT, alanine aminotransferase; AST, Aspartate aminotransferase, BMI, body mass index; DBP, diastolic blood pressure; $\gamma$ GT, Gamma-glutamyl transpeptidase; HDL, high density lipoprotein; LDL, low density lipoprotein; SBP, systolic blood pressure. 
Table 2. Demographic and metabolic characteristics by infection status of individuals studied in the clinic.

\begin{tabular}{|c|c|c|c|c|c|c|c|c|}
\hline & \multicolumn{2}{|c|}{ Never infected $(n=192)$} & \multicolumn{3}{|c|}{ Cleared infection $(n=115)$} & \multicolumn{3}{|c|}{ Chronic infection $(\mathrm{n}=187)$} \\
\hline Variable & Mean or $\%$ & $95 \% \mathrm{CI}$ & Mean or $\%$ & $95 \% \mathrm{CI}$ & $\mathrm{p}$ value & Mean or $\%$ & $95 \% \mathrm{CI}$ & $\mathrm{p}$ value \\
\hline Age (years) & 50.2 & $48.6,51.7$ & 51.0 & $49.3,52.7$ & 0.5 & 50.8 & $49.7,51.9$ & 0.5 \\
\hline Male \% (n) & $45(87)$ & & $51(59)$ & & 0.3 & $63(118)$ & & 0.001 \\
\hline BMI, $\mathrm{kg} / \mathrm{m}^{2}$ & 29.1 & $28.3,29.9$ & 28.9 & $27.9,29.9$ & 0.8 & 27.7 & $26.9,28.5$ & 0.01 \\
\hline Fat $\%$ & 30.6 & $29.5,31.7$ & 30.5 & $29.1,31.9$ & 0.9 & 29.1 & $28.0,30.2$ & 0.05 \\
\hline Waist hip ratio (men) & 0.91 & $0.90,0.93$ & 0.92 & $0.90,0.94$ & 0.6 & 0.92 & $0.90,0.93$ & 0.7 \\
\hline Waist hip ratio (women) & 0.88 & $0.87,0.89$ & 0.86 & $0.85,0.88$ & 0.2 & 0.86 & $0.84,0.88$ & 0.09 \\
\hline Mesenteric fat (mm) & 32.7 & $31.0,34.4$ & 37.8 & $35.6,40.0$ & $<0.0001$ & 36.4 & $34.5,38.2$ & 0.004 \\
\hline Subcutaneous fat $(\mathrm{mm})$ & 12.4 & $11.8,13.0$ & 11.5 & $10.8,12.3$ & 0.08 & 11.1 & $10.4,11.7$ & 0.004 \\
\hline SBP, mmHg & 122 & 119,125 & 119 & 115,122 & 0.1 & 119 & 116,122 & 0.1 \\
\hline $\mathrm{DBP}, \mathrm{mmHg}$ & 76 & 74,77 & 72 & 70,75 & 0.03 & 74 & 72,75 & 0.1 \\
\hline Total cholesterol, mmol/l & 5.16 & $5.00,5.32$ & 5.28 & $5.06,5.50$ & 0.4 & 4.36 & $4.20,4.53$ & $<0.0001$ \\
\hline LDL-cholesterol, mmol/1 & 3.45 & $3.30,3.60$ & 3.56 & $3.34,3.78$ & 0.4 & 2.69 & $2.53,2.86$ & $<0.0001$ \\
\hline Glucose, $\mathrm{mmol} / \mathrm{l}^{*}$ & 4.83 & $4.68,4.99$ & 4.95 & $4.76,5.19$ & 0.3 & 5.01 & $4.86,5.48$ & 0.2 \\
\hline Insulin $\mu \mathrm{IU} / \mathrm{ml}^{*}$ & 4.00 & $3.42,4.66$ & 3.99 & $3.24,4.91$ & 0.9 & 5.81 & $4.85,6.95$ & 0.004 \\
\hline HOMA-IR* & 0.50 & $0.42,0.60$ & 0.50 & $0.40,0.63$ & 1.0 & 0.73 & $0.60,0.89$ & 0.005 \\
\hline HOMA-S* & 198 & 167,237 & 200 & 159,252 & 1.0 & 137 & 112,167 & 0.005 \\
\hline HOMA-B* & 59 & 52,67 & 57 & 48,68 & 0.8 & 73 & 63,86 & 0.04 \\
\hline C-peptide & 1.26 & $1.07,1.48$ & 1.43 & $1.13,1.80$ & 0.4 & 1.31 & $1.09,1.57$ & 0.7 \\
\hline AST, IU/L* & 21.8 & $20.4,23.2$ & 20.9 & $19.3,22.8$ & 0.5 & 35.1 & $32.8,33.3$ & $<0.0001$ \\
\hline Diabetes & 4.6 & $1.8,7.5$ & 9.6 & $4.0,14.2$ & 0.1 & 7.0 & $3.3,11.1$ & 0.4 \\
\hline Hypertension & 21.0 & $15.3,26.7$ & 15.7 & $9.8,21.6$ & 0.3 & 16.2 & $11.0,21.5$ & 0.2 \\
\hline Current smoker & 5.8 & $2.6,9.1$ & 9.6 & $4.4,14.9$ & 0.2 & 14.2 & $9.1,19.2$ & 0.005 \\
\hline
\end{tabular}


Apart from age and sex themselves, all other parameters are adjusted (continuous) or standardised (categorical) for age and sex (except waist hip ratio, where age adjusted values are presented stratified by sex).

*Log transformed variable.

Abbreviations: AST, Aspartate aminotransferase, BMI, body mass index; DBP, diastolic blood pressure; LDL, low density lipoprotein; SBP, systolic blood pressure. 
Table 3. Mean carotid IMT (mm), and burden of carotid plaque by infection status

\begin{tabular}{|c|c|c|c|c|c|c|c|c|}
\hline \multirow[b]{2}{*}{ Carotid IMT (mm) } & \multicolumn{2}{|c|}{ Never infected (192) } & \multicolumn{2}{|c|}{ Cleared infection (115) } & \multirow[t]{2}{*}{$\begin{array}{c}\mathbf{p} \\
\text { value }\end{array}$} & \multicolumn{2}{|c|}{$\begin{array}{c}\text { Chronic infection } \\
\text { (187) }\end{array}$} & \multirow[t]{2}{*}{ p value } \\
\hline & Mean & $95 \% \mathrm{CI}$ & Mean & $95 \% \mathrm{CI}$ & & Mean & $95 \% \mathrm{CI}$ & \\
\hline Adjusted for age and sex & 0.71 & $0.68,0.74$ & 0.71 & $0.66,0.75$ & 0.9 & 0.73 & $0.70,0.76$ & 0.4 \\
\hline Fully adjusted & 0.70 & $0.67,0.73$ & 0.69 & $0.65,0.74$ & 0.8 & 0.76 & $0.72,0.79$ & 0.03 \\
\hline
\end{tabular}

\section{Carotid plaque}

$\mathrm{N}(\%)$

$10(5.2 \%)$

$4(3.5 \%)$

$12(6.4 \%)$

Odds ratio, adjusted for

age and sex

1.00

Odds ratio, fully adjusted

1.00

$0.64 \quad 0.21,1.98$

$0.32 \quad 0.06,1.64$

0.4

0.2

\section{$1.88 \quad 0.77,4.56$}

$3.49 \quad 1.22,9.97$

\section{$P$ values compare either cleared or chronic infection group with never infected.}

Comparisons of odds ratios for plaque use the never infected group as the comparator (odds ratio $=1.00$ )

The fully adjusted model was adjusted for age, sex, systolic blood pressure, diabetes, fasting glucose, BMI, low density lipoprotein cholesterol, and smoking status. 
